\title{
Simulated loss of foveal vision eliminates visual search advantage in repeated displays
}

\section{Franziska Geringswald ${ }^{1}$, Florian Baumgartner ${ }^{1}$ and Stefan Pollmann ${ }^{1,2}$ *}

${ }^{1}$ Department of Experimental Psychology, University of Magdeburg, Magdeburg, Germany

${ }^{2}$ Center for Behavioral Brain Sciences, Magdeburg, Germany

\section{Edited by:}

Hans-Jochen Heinze, University of Magdeburg, Germany

Reviewed by:

Árni Kristjánsson, University of Iceland, Iceland

Hermann Josef Mueller, University of Munich, Germany

\section{${ }^{*}$ Correspondence:}

Stefan Pollmann, Institut für

Psychologie II, Otto-von-Guericke-

Universität Magdeburg, Postfach

4120, D-39016 Magdeburg, Germany.

e-mail: stefan.pollmann@ovgu.de
In the contextual cueing paradigm, incidental visual learning of repeated distractor configurations leads to faster search times in repeated compared to new displays. This contextual cueing is closely linked to the visual exploration of the search arrays as indicated by fewer fixations and more efficient scan paths in repeated search arrays. Here, we examined contextual cueing under impaired visual exploration induced by a simulated central scotoma that causes the participant to rely on extrafoveal vision. We let normal-sighted participants search for the target either under unimpaired viewing conditions or with a gaze-contingent central scotoma masking the currently fixated area. Under unimpaired viewing conditions, participants revealed shorter search times and more efficient exploration of the display for repeated compared to novel search arrays and thus exhibited contextual cueing. When visual search was impaired by the central scotoma, search facilitation for repeated displays was eliminated. These results indicate that a loss of foveal sight, as it is commonly observed in maculopathies, e.g., may lead to deficits in high-level visual functions well beyond the immediate consequences of a scotoma.

Keywords: contextual cueing, visual search, visual attention, visual memory, artificial scotoma

\section{INTRODUCTION}

Attending to a particular part of the environment can be guided by regularities in the environment, which are often not consciously perceived. To exploit such regularities is clearly adaptive, because it enables us to find stimuli more efficiently than by a random spatial search (for a recent review, see Kristjánsson and Campana, 2010). Repetition of the spatial layout of a scene can facilitate finding the target location. This has consistently been reported in investigations of the contextual cueing effect (first reported by Chun and Jiang, 1998). The core finding is that target detection is facilitated if the spatial arrangement of distractor stimuli is repeated across experimental blocks compared to search for the same target in a new distractor arrangement. This effect may occur although participants are unable to recognize the distractor repetition. Thus, memory for spatial locations, gained by incidental learning, increases the speed of target detection in visual search, an effect that has been observed as long as 1 week after training (Chun and Jiang, 2003). Facilitation in target detection in repeated distractor layouts has been argued to operate through improvement of attentional guidance to the target location using reaction time measures in inefficient serial search (Kunar et al., 2008) as well as accuracy measures in efficient pop-out search (Geyer et al., 2010b) provided that enough time is given to encode the search arrays and develop guidance. Additionally, post-selective processes of response selection at a later stage of processing might be enhanced by contextual cueing (Kunar et al., 2007).

Here, we investigated whether contextual cueing will be affected by the presence of a central scotoma. This research question was inspired by the characteristic retinal pathology of patients with age-related macular degeneration (AMD), in which the macula, the region with the highest density of photoreceptors, degenerates and causes a severe loss of central vision at advanced states of the disease (Lindblad et al., 2009). Thus, the patients are forced to rely on extrafoveal vision for all processes typically carried out in foveal vision. This extrafoveal vision has lower spatial resolution compared to foveal vision. Many studies on low-level visual processing have illustrated the immediate consequences of the loss of foveal vision, e.g., reduced visual acuity and contrast sensitivity (Qiu and Leat, 2009) and deficits in orientation discrimination (Bedell et al., 2009) and shape discrimination (Wang et al., 2002). For a review on psychophysical function in AMD, see Neelam et al. (2009).

Loss of foveal vision may also make the visual exploration of the environment with eye-movements more difficult. While some studies found no influence of a central scotoma on eye-movement parameters during visual search (Murphy and Foley-Fischer, 1988; Shen et al., 2003), other studies found that visual search for highacuity targets could not be compensated efficiently by, e.g., longer fixation durations, leading to increased search times (Bertera, 1988; Cornelissen et al., 2005).

However, the loss of acuity is not the only potential problem affecting visual search with a central scotoma. Eye-movements are intimately linked to attention (Hoffman and Subramaniam, 1995; Deubel and Schneider, 1996). During visual search, attention is often attracted by extrafoveal input (either salient stimuli or features matching the target template), leading to a subsequent fixation of this location. The tendency to fixate a target in order to process it attentively is so dominant, that it has been observed even in the presence of a simulated foveal scotoma, where it is clearly counterproductive (Miellet et al., 2010). This tendency is so stable 
that it took patients with central scotoma 20 months or more to overcome it (Timberlake et al., 1986) leading to the question whether attentional processing of the target (and its surrounding) becomes compromised in the presence of a central scotoma. This, in turn, may lead to deficient visual memory, because fixating an object is closely tied to its later recall (Hollingworth, 2006). It may also interfere with the use of implicitly learned context cues for efficient visual search that has been shown to depend on selective spatial attention and visuospatial working memory (Jiang and Leung, 2005; Manginelli et al., 2011). Furthermore, search with a simulated central scotoma will very likely lead to an increase of top-down controlled search strategies, until the participants get accustomed to the presence of the scotoma. This may also interfere with search facilitation in repeated displays, as has been shown previously in a comparison of search under stimulus-driven vs. top-down controlled search instructions (Lleras and von Mühlenen, 2004). Additionally, controlling eye-movements with an artificial scotoma might be so demanding in terms of cognitive load that only insufficient capacity might be available for peripheral processing of the spatial arrangement of the search items. High cognitive load in dual tasks has been shown to increase distractor interference (Lavie et al., 2004) and thus more top-down controlled search strategies with an artificial scotoma might interfere with the deployment of selective attention to the target in repeated search arrays.

Thus, there are a number of reasons why a central scotoma may lead to reduced search efficiency in familiar environments, an effect that transcends the immediate consequences of the scotoma and may be of importance for patients suffering from a central scotoma. As a first step, we investigated whether the search facilitation in repeated search displays, which is reliably obtained in normal participants, is reduced in the presence of a central scotoma. It is beyond the scope of this paper to discriminate between the possible causes of reduced search efficiency but we will discuss potential future studies toward this end.

We simulated a central scotoma with gaze-contingent displays in normal-sighted participants (moving mask paradigm, e.g., Rayner and Bertera, 1979). This enabled us to investigate the effect of a well-defined scotoma in isolation that is problematic in typical AMD patients as their visual deficits are often highly variable or interact with additional visual or cognitive impairments due to their age. It should be noted that the current study, while it may lead to hypotheses concerning contextual cueing in AMD, does not eliminate the need to carry out AMD-patient studies to investigate this issue.

Contextual cueing leads not only to reduced search times, but also to a reduced number of fixations (Peterson and Kramer, 2001; Tseng and Li, 2004; Brockmole and Henderson, 2006; Manginelli and Pollmann, 2009) and more efficient scan paths (Tseng and Li, 2004; Brockmole and Henderson, 2006; Manginelli and Pollmann, 2009). Because of the interference of the central scotoma with display exploration, we expected selectively reduced search facilitation in repeated displays after sufficient learning, in comparison with search in novel displays. In contrast, the search facilitation with increasing practice that is typically observed for repeated and novel displays alike, due to automatization of search routines, should be unaffected by a scotoma.

\section{MATERIALS AND METHODS PARTICIPANTS}

Thirty healthy participants ( 3 males, 27 females, 25 years average age) participated in the experiment. All participants reported normal or corrected to normal visual acuity and were not informed about the purpose of the study until they had completed the two experimental sessions. The experiments were approved by the Ethics Committee of the University of Magdeburg. Informed written consent was obtained prior to the experiments. Participants were compensated with course credits or received a compensation of Euro 12.

\section{APPARATUS}

Stimulus presentation and response recording were controlled using the Psychtoolbox (Brainard, 1997; Pelli, 1997) under Matlab on a PC with a $22^{\prime \prime}$ Iiyama Vision Master Pro 511 CRT monitor. The monitor was $400 \mathrm{~mm}$ (1280 pixels) wide and $300 \mathrm{~mm}$ (960 pixels) high and the vertical refresh rate was $100 \mathrm{~Hz}$. Participants viewed the stimuli binocularly from a distance of $60 \mathrm{~cm}$ leading to a pixel size of $0.03^{\circ}$ or 1.79 arcminute of visual angle. Eye position was recorded using an iViewX Hi-Speed eye tracking system (SensoMotoric Instruments GmbH, Teltow, Germany), with a temporal resolution of $240 \mathrm{~Hz}$. The gaze data was filtered by the heuristic (stage 1) and the bilateral filters implemented in the iView X software. Head movements were minimized by stabilizing participants' head using a chin- and forehead rest. Participants were tested individually in a dimly lit, sound-attenuated chamber.

\section{STIMULI}

Each search display was composed of 1 target $\left(90^{\circ}\right.$ or $270^{\circ}$ rotated $\mathrm{T})$ and 11 distractors $\left(0^{\circ}, 90^{\circ}, 180^{\circ}, 270^{\circ}\right.$ rotated $\left.\mathrm{L}\right)$ with each item subtending $1.04^{\circ} \times 1.04^{\circ}$ (Figure 1). The orientation of the target was randomly chosen for each trial and did therefore neither correlate with the specific displays nor the experimental conditions. The items were black presented on a gray background. Following results from pilot experiments, an offset of $0.12^{\circ}$ was introduced at the junction of the two segments of the L-shapes to increase search difficulty. The stimulus positions were randomly chosen on four imaginary concentric circles with radii of $2.03^{\circ}, 4.74^{\circ}, 7.43^{\circ}$, and $10.15^{\circ}$ each containing $4,12,20$, and 28 equidistant possible item locations respectively. The target positions were defined on the two intermediate circles. For each participant, 24 target positions were randomly chosen before the beginning of the experiment. For 12 of those target positions, randomly assigned distractor configurations remained constant throughout the experiment ("repeated" condition). The other 12 target positions were combined with randomly generated distractor configurations for each experimental block ("novel" condition). The target and distractor positions were balanced across quadrants and conditions. All search items were equally distributed across the display in each trial, i.e., each quadrant of the display always contained three search items. The chosen stimulus coordinates on the circles defined the circumcenter of the search items. Thus, the overall size of the search display on the screen extended a circular area with a diameter of $21.34^{\circ}$.

The simulated scotoma was created as a circular area with a diameter of $7^{\circ}$ visual angle which completely covered the visual 


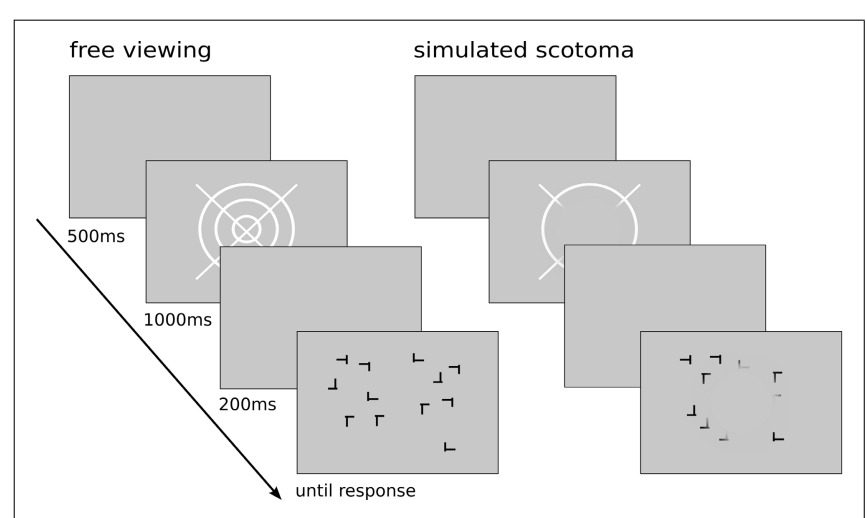

FIGURE 1 | Schematic diagram of an experimental trial. A trial consisted of the presentation of a blank screen $(500 \mathrm{~ms})$, a fixation target (1000 ms), another blank screen $(200 \mathrm{~ms}$ ), and the search display (presented until response). Left: A trial in the unimpaired condition. Right: Visualization of the display layout with the simulated scotoma covering parts of the screen. The scotoma had a diameter of $9^{\circ}$ smoothly fading out at the edges. The fully opaque area of the scotoma measured $7^{\circ}$ in diameter which appeared invisible to the participant while the visibility of the stimulus display gradually increased at the edge of the opaque area.

stimulus. At the border of the scotoma the transparency gradient followed the increasing slope of a Gaussian distribution with a deviation of $1^{\circ}$ visual angle. After rendering the patch with the Gaussian filter, a concentric disk with a diameter of $7^{\circ}$ within the alpha channel was set to $100 \%$. Thus, the fully opaque size of the scotoma extended $7^{\circ}$ smoothly fading out at the edges. The scotoma was superimposed on the search display and colored the same gray as the background. Therefore the scotoma could only be perceived by the participant when it covered a stimulus patch of a distinct color as the masked part of the display became invisible to the participant, thus simulating the negative effect of a central scotoma. The position of the scotoma was updated with the gaze coordinates retrieved from the iViewX Hi-Speed tracking system with an estimated delay of maximal $10 \mathrm{~ms}$ due to the heuristic filtering of the gaze samples. The estimated worst-case latency until the update of the display was two frames on the CRT (20 $\mathrm{ms}$ ), adding up to a total of a maximum delay of the scotoma of $30 \mathrm{~ms}$. Before running the contextual cueing experiments with the artificial scotoma, we validated the simulation by means of a behavioral visual discrimination task which required foveal processing of high-acuity target stimuli. This experiment confirmed that the scotoma simulation was properly implemented in technical terms as the scotoma severely impaired foveal processing of target stimuli leading to a highly significant decrease of discrimination performance with the scotoma compared to almost perfect discrimination when targets could be foveated. The manuscript of this validation experiment is currently under review.

The fixation stimulus was designed as a white target consisting of three concentric circles and two diagonal line segments intersecting at the center and subtending an area of $9^{\circ} \times 9^{\circ}$.

\section{PROCEDURE}

Participants searched for a left- or right tilted $\mathrm{T}$ among Lshaped distractors and indicated its orientation with left and right mouse button presses with the right hand. Each participant completed one experimental session with and one without the gaze-contingent scotoma within a period of approximately 1 week. The order of the sessions was randomized across participants and a set of distinct old displays was generated for each session. Each session started with a 13-point gaze calibration, followed by a short training period to familiarize participants with the task, the actual search experiment and a recognition test. In the scotoma session, the simulated central scotoma was present throughout all tasks except when instructions were presented on the screen.

The training was composed of 24 trials with randomly generated displays containing target positions that were not used in the experimental configurations. The search experiment consisted of 20 blocks of 24 trials each. Every trial started with the presentation of a blank for $500 \mathrm{~ms}$ followed by the fixation stimulus for $1000 \mathrm{~ms}$. Another blank was shown for $200 \mathrm{~ms}$ before the search display was presented. The search display remained on the screen until the participant responded (Figure 1). Auditory feedback was provided for correct (a 2000-Hz high-pitch tone) and wrong answers (a 500-Hz low-pitch tone). Participants were instructed to search for the target $\mathrm{T}$ as fast and accurately as possible. They were further asked to follow their intuition and search for the target $\mathrm{T}$ passively as suggested by Lleras and von Mühlenen (2004). In the session with the simulated scotoma participants were asked to try to place the gray patch at the center of the fixation target in order to equalize starting positions in the search task.

The recognition test included the original 12 repeated and another 12 new randomly generated configurations presented in randomized order. Participants were asked to indicate whether they had seen the displays during the course of the experiment or not by mouse button presses. No feedback about the correctness of the answer was given. Participants started each block by pressing a key, allowing for self-terminated breaks between the blocks. One session lasted approximately $1 \mathrm{~h}$.

\section{GAZE DATA ANALYSIS}

Gaze data was analyzed by a custom-made Python script using a velocity-based algorithm. We used the procedures described in Nyström and Holmqvist (2010) for filtering the data with the Savitzky-Golay FIR smoothing filter (Savitzky and Golay, 1964) algorithm with second-order polynomials and a filter length of $20 \mathrm{~ms}$, the removal of invalid gaze samples due to eye blinks or signal losses and the calculation of adaptive velocity thresholds. We then identified saccade events by velocities that exceeded the adaptive peak velocity threshold. The saccade start was calculated as the first sample before the velocity peak that fell above the individual saccade velocity threshold and after which the velocity of the eye increased monotonically. The saccade end was calculated as the closest sample after the velocity peak that fell below the individual saccade velocity threshold and was the last sample of monotonically decreasing velocity. Fixations were then identified from the inter-saccadic events that exceeded a duration of $100 \mathrm{~ms}$.

Two dependent measures were calculated from the fixation events. First, we measured the number of fixations required to 
find the target stimulus from the onset of the search display until the mouse button was pressed. The second measure of interest was the efficiency of the scan path toward the target which was calculated as the ratio between the total distance covered by the eye during the search for the target, i.e., the sum of distances between each consecutive fixation, and the shortest path possible, i.e., the distance between the first fixation and the target location (Brockmole and Henderson, 2006). Thus, more direct scan paths toward the target position are characterized by lower scan pattern ratios.

\section{DATA EXCLUSION}

We applied three data exclusion criteria for the analysis of search times and gaze parameters. First, we removed all erroneous responses from the data set. Participants whose average performance in the discrimination task was below the average minus two times the standard deviation of all participants' performance in at least one viewing condition were excluded. This criterion led to the exclusion of two participants. The first participant exhibited a mean performance of $90.4 \%$ in the unimpaired viewing and $82.3 \%$ in the scotoma condition and the second participant a mean performance of $94.0 \%$ in the unimpaired and $81.0 \%$ in the scotoma condition. As the reliable presentation of the scotoma during search depended on the availability of the gaze coordinates retrieved from the eye-tracker, we furthermore decided to remove search trials in which more than $20 \%$ of the gaze samples were missing due to signal losses. Third, we excluded trials in which the search time was shorter than 200 ms or longer than two standard deviations than the participant's average search time in the remaining trials.

After removing all invalid data we calculated the overall percentage of data excluded for each participant and each experimental session and identified data sets in which more than $20 \%$ of all trials were classified as invalid. This method led to the exclusion of three further participants: the first participant's data in the unimpaired condition contained $28.3 \%$ of invalid data, the second participant's data $24.0 \%$ in the scotoma condition and the third participant's data $32.1 \%$ in the scotoma condition. The two latter steps of preprocessing were also applied before accuracy analysis. Thus, 25 participants were included in all further analyses. The average percentage of invalid noise trials was 3.8\% $(\mathrm{SD}=4.6 \%)$ in the unimpaired condition and $3.0 \%(\mathrm{SD}=3.3 \%)$ in the scotoma condition. Search time outliers amounted to $1.7 \%(\mathrm{SD}=0.6 \%)$ of the data in the unimpaired condition and $1.7 \%(\mathrm{SD}=0.5 \%)$ in the scotoma condition.

In summary, all three steps of the exclusion procedure led to the rejection of $6.0 \%(\mathrm{SD}=4.8 \%)$ of invalid data in the unimpaired condition and $7.3 \%(\mathrm{SD}=3.7 \%)$ in the scotoma condition. There was no difference in the amount of data excluded for the unimpaired vs. the scotoma condition $[t(24)=-1.320$, $p=0.200]$.

\section{RESULTS}

\section{ACCURACY}

The accuracies ranged from 94.0 to $100.0 \%$ (average $98.3 \%$ ) in the unimpaired condition and from 90.2 to $99.6 \%$ (average $96.9 \%$ ) in the scotoma condition. In order to test for differences in accuracies concerning the viewing conditions an ANOVA on errors with the factors viewing condition (no scotoma vs. scotoma) and configuration (repeated vs. novel) was carried out. It revealed a significant main effect of viewing condition $[F(1,24)=11.075, p<0.05]$ reflecting less accurate performance when vision was impaired by the artificial scotoma. Neither the main effect of configuration $[F(1,24)=2.178, p=0.153]$ nor the interaction $[F(1,24)=0.123$, $p=0.730]$ were significant.

\section{SEARCH TIMES}

Experimental blocks were aggregated to four epochs, each containing five blocks, in order to increase power. Averaged search times for the four epochs for repeated and novel displays separated by viewing condition can be seen in Figure 2. In a first step, two separate two-way, repeated measures analyses of variance (ANOVAs) for the two experimental conditions with the factors configuration (repeated, novel) and epoch $(1,4)$ were performed on search times. We decided to compare only the first and the last epoch of the search experiment to increase the power in order to detect differences at the beginning of the session, when learning effects should be small, and at the end of the session, when contextual cueing should be most prominent. The mean differences between repeated and novel displays for all epochs are reported in Table 1 . In the control condition, the main effects of epoch $[F(1,24)=36.700, p<0.05]$ and configuration $[F(1,24)=19.190, p<0.05]$ were significant. The interaction of configuration and epoch was also significant $[F(1,24)=8.582$, $p<0.05$ ]. Thus, in addition to a general improvement of search speed over time, a robust advantage for repeated displays (i.e., a contextual cueing effect) developed over the course of the experiment.

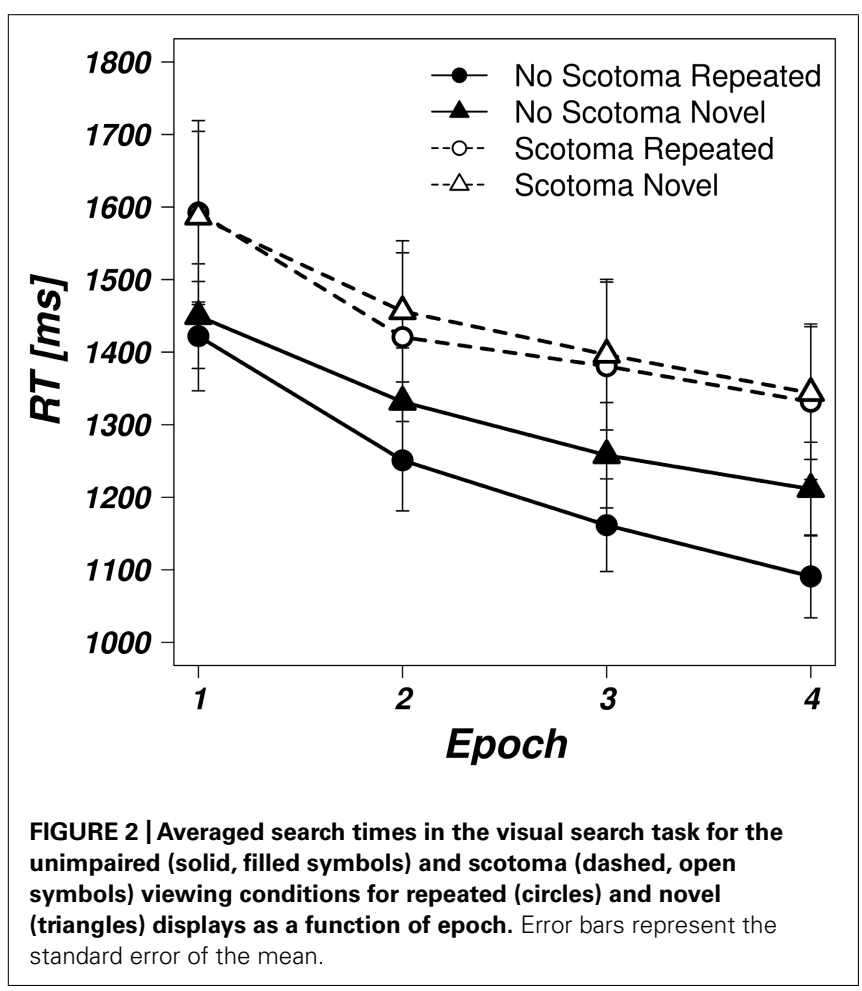


Table 1 | Mean difference between novel and repeated configurations.

\begin{tabular}{|c|c|c|c|c|c|}
\hline & \multirow[t]{2}{*}{ Condition } & \multicolumn{4}{|c|}{ Mean difference novel-repeated } \\
\hline & & Epoch 1 & Epoch 2 & Epoch 3 & Epoch 4 \\
\hline \multirow[t]{2}{*}{ Search time (ms) } & Control & 27.58 & 80.60 & 96.44 & 120.43 \\
\hline & Scotoma & -5.70 & 35.54 & 15.99 & 12.03 \\
\hline Number of fixations & Control & 0.16 & 0.26 & 0.35 & 0.44 \\
\hline (units) & Scotoma & 0.05 & 0.12 & 0.10 & 0.15 \\
\hline Scan pattern ratio & Control & 0.18 & 0.29 & 0.33 & 0.55 \\
\hline (units) & Scotoma & -0.24 & 0.21 & -0.12 & 0.05 \\
\hline
\end{tabular}

In the scotoma condition, there was also a general improvement in search times over time as revealed by a significant main effect of epoch $[F(1,24)=18.900, p<0.05]$. However, neither the main effect of configuration $[F(1,24)=0.015, p=0.902]$ nor the interaction $[F(1,24)=0.166, p=0.688]$ reached significance indicating that the simulated scotoma interfered with contextual cueing.

Next, we were specifically interested in search performance in the last epoch of the session. We compared participants' search times by means of a two-way, repeated measures ANOVA with the factors viewing condition (no scotoma, scotoma) and display type (repeated, novel). The main effect of viewing condition was significant $[F(1,24)=5.748, p<0.05]$ with higher search times in the scotoma (mean $=1338 \mathrm{~ms}$ ) than in the control condition (mean $=1151 \mathrm{~ms}$ ). The main effect of display type did also reach significance $[F(1,24)=12.640, p<0.05]$ with an average advantage of $66 \mathrm{~ms}$ in repeated displays. Additionally, the interaction between viewing condition and display type was significant $[F(1,24)=5.711, p<0.05]$ indicating that the search facilitation for repeated displays was reduced when search was impaired by the scotoma. While search times for repeated and novel displays were similar in the scotoma condition (1332 vs. $1344 \mathrm{~ms}$ respectively), repeated displays speeded up search in the control condition by $120 \mathrm{~ms}$. This indicates that at the end of the sessions, after participants had been able to get used to the search with the scotoma, the visual impairment was still affecting search efficiency. In addition, search facilitation for repeated displays was reduced in search with the scotoma compared to the control condition.

\section{GAZE}

\section{Number of fixations}

Figure 3 depicts the mean number of fixations for repeated and novel displays in the unimpaired and the scotoma condition. Analogous to search times, participants needed less fixations in order to find the target over the course of the experiment in the unimpaired condition as revealed by a significant main effect of the factor epoch $[F(1,24)=38.406, p<0.05]$. Furthermore, a significant main effect of display type $[F(1,24)=21.101, p<0.05]$ was observed with less fixations in the repeated displays. The interaction between epoch and display type was also significant $[F(1,24)=6.477, p<0.05]$, indicating that contextual learning lead to more efficient search strategies requiring less fixations for repeated displays.

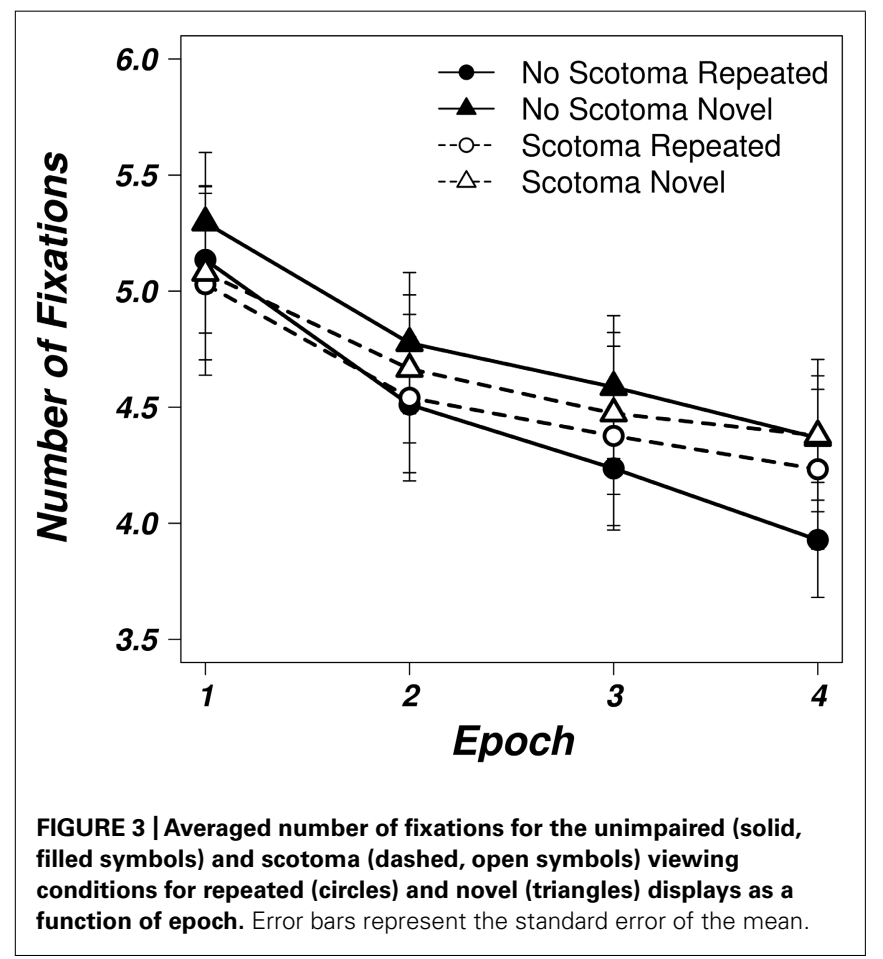

In the scotoma condition, we could only observe a significant main effect of epoch $[F(1,24)=17.582, p<0.05]$ which indicates that participants were able to find more efficient search strategies after repeatedly searching through the stimulus arrays with the scotoma. However, neither the main effect of display type $[F(1,24)=1.363, p=0.255]$ nor the interaction $[F(1,24)=0.420$, $p=0.523]$ were significant, thus the amount of fixations necessary to find the target in random and repeated search arrays did not differ.

Additionally, we examined the number of fixations of the last epoch under impaired and unimpaired search with a two-way, repeated measures ANOVA with the factors viewing condition (no scotoma, scotoma) and display type (repeated, novel). The main effect of viewing condition was not significant $[F(1,24)=0.393$, $p=0.537]$ revealing that the average number of fixations required to find the target was equal for the control (mean $=4.15)$ and the scotoma condition ( mean $=4.31)$. The main effect of display type reached significance $[F(1,24)=21.190, p<0.05]$. In repeated displays the target was detected after an average of 4.08 fixations whereas 4.37 fixations were necessary in random displays. The interaction between viewing condition and display type did not reach significance $[F(1,24)=3.531, p=0.072]$. When searching with the scotoma, observers needed 4.38 fixations on average for novel and 4.23 fixations for repeated displays. Without the scotoma, the average number of fixations was 4.37 in novel displays and 3.93 in repeated displays.

\section{Scan pattern ratio}

The development of average scan pattern ratios for repeated and novel displays in the two viewing conditions can be seen in Figure 4. A repeated measures ANOVA on the factors display type and epoch revealed a significant main effect of the factor 


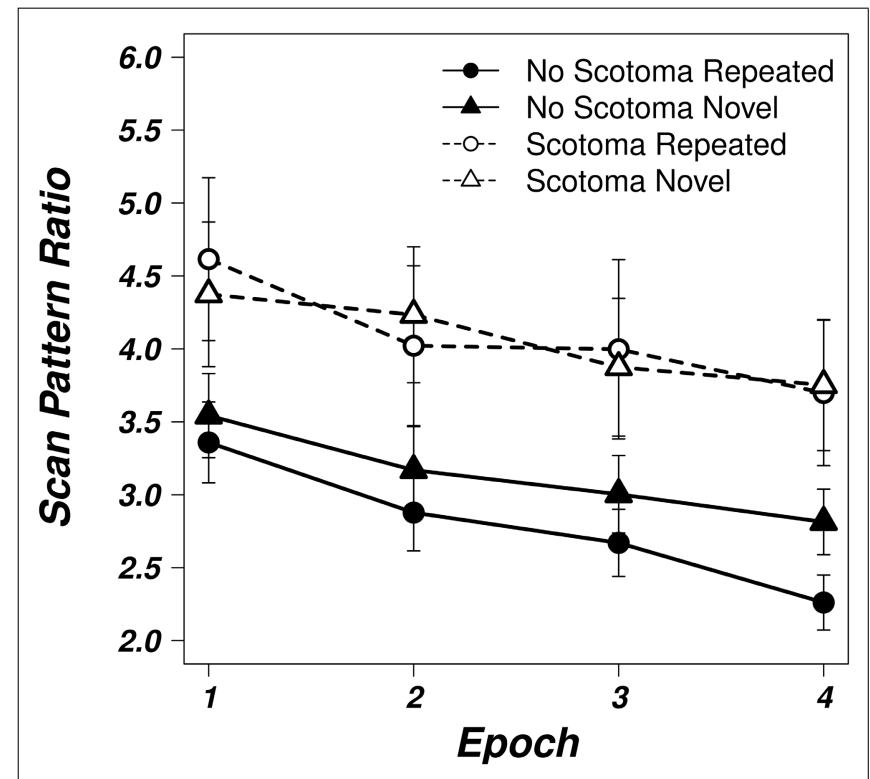

FIGURE 4 | Averaged scan pattern ratios for the unimpaired (solid, filled symbols) and scotoma (dashed, open symbols) viewing conditions for repeated (circles) and novel (triangles) displays as a function of epoch. Error bars represent the standard error of the mean.

epoch $[F(1,24)=30.156, p<0.05]$, a significant main factor of display type $[F(1.24)=17.478, p<0.05]$, and a significant interaction $[F(1.24)=7.201, p<0.05]$ in the unimpaired viewing condition. Thus, scan paths became not only more efficient with repeated search for the target, but repeated displays additionally revealed more direct scan paths than random search arrays.

In the scotoma condition, scan paths shortened over time as shown by a significant main effect of epoch $[F(1,24)=11.524$, $p<0.05]$, but repeated displays did not show an additional advantage as neither the main effect of display type $[F(1,24)=0.608$, $p=0.443]$ nor the interaction $[F(1,24)=1.104, p=0.304]$ reached significance.

Analogous to the analyses above, we carried out a two-way, repeated measures ANOVA with the factors viewing condition (no scotoma, scotoma) and display type (repeated, novel) on scan pattern ratios of the last epoch of the experiment. The main effect of viewing condition was significant $[F(1,24)=9.670$, $p<0.05$ ] showing that the average scan paths were less efficient when search was impaired by the scotoma. The scan paths were 3.72 times the direct distance from the first fixation to the target in the scotoma condition and 2.54 times the direct distance in the control condition. The main effect of display type did also reach significance $[F(1,24)=6.412, p<0.05]$ with an average scan pattern ratio of 2.98 in repeated displays and 3.28 in novel displays. Additionally, the interaction between viewing condition and display type was significant $[F(1,24)=4.393$, $p<0.05]$. When search was impaired by the scotoma, the scan pattern ratios for repeated and novel displays were nearly identical. In repeated displays, the eye of the participants moved on average 3.75 times the most direct line compared to 3.70 times in repeated displays. In the control condition, the scan pattern ratio improved from 2.81 in novel displays to 2.26 in repeated search arrays.

\section{RECOGNITION TEST}

Mean recognition accuracy in the unimpaired condition was $57.17 \%(\mathrm{SD}=10.10 \%)$. Participants correctly classified repeated displays as old (the hit rate) on $57.33 \%(\mathrm{SD}=21.29 \%$ ) of trials and falsely reported $43.0 \%(\mathrm{SD}=15.33 \%)$ of novel displays as old (the false alarm rate). A paired $t$-test on the hit and false alarm rates reached significance $[t(24)=3.548, p<0.05]$. In order to test for a correlation between the size of the contextual cueing effect and recognition accuracy we calculated the individual amount of search facilitation for repeated displays for each subject in the last epoch. This was done by calculating the difference in mean reaction times between the novel and the repeated displays. We then divided these absolute differences by the mean reaction times of novel displays in order to standardize the contextual cueing effects. The normalized magnitude of contextual cueing did not correlate with the recognition accuracy $(r=-0.203$, $p=0.331$ ).

Mean recognition accuracy in the scotoma condition was $55.33 \%(\mathrm{SD}=11.10 \%)$ with a mean hit rate of $49.0 \%(\mathrm{SD}=$ $19.88 \%)$ and a mean false alarm rate of $42.33 \%(\mathrm{SD}=16.30 \%)$. A paired $t$-test on hit and false alarm rates did not reach significance $[t(24)=1.503, p=0.146]$. The normalized magnitude of contextual cueing correlated positively with the recognition accuracy $(r=0.412, p<0.05)$.

\section{DISCUSSION}

Does the loss of foveal vision affect efficient search in repeated environments? To answer this question, we investigated the impact of a simulated central scotoma on contextual cueing effects in visual search. The central finding was that visual search with a scotoma eliminated the search facilitation in repeated displays that was observed, in agreement with many previous studies, in search without a scotoma. This elimination of search facilitation in search with a scotoma was observed in search times as well as in eye gaze parameters such as number of fixations and scan pattern ratios. Thus, while in non-scotomatous search, the repeated displays could be used to speed up overall search time by more efficient scanning of the display, this was not the case with a simulated scotoma.

Visual search was generally slower with the simulated scotoma than without, reflecting the unusual need to use exclusively peripheral vision to explore the search display. We observed comparable improvements of search times and gaze parameters over the course of the experiment with and without scotoma. Thus, search with a scotoma benefitted from training, but was still less efficient than unimpaired search at the end of the experiment. Nevertheless, participants performed reasonably well at the search task as indicated by accuracy rates well above 95\% and search times below $1.5 \mathrm{~s}$ in the scotoma condition. Thus, the search displays were well designed in order to investigate peripheral search behavior.

Noteworthy, search with a scotoma did not require more fixations in order to find the target. We did not expect this, but, 
in hindsight, it is perhaps not surprising that search with a simulated scotoma did not lead to an increase of fixations compared with unimpaired search. This may reflect the need to control the exploration of the search displays in a more topdown controlled manner when a scotoma is simulated than in its absence, making each fixation more "costly" in terms of programming.

This top-down controlled search, however, was not as efficient as the search without scotoma. Other than for number of fixations, there was not only a clear advantage for repeated, but also for novel displays searched without scotoma. The scan paths for random displays were about 3.8 times longer than the most direct path to the target from the first fixation in the presence of a scotoma whereas they were only 2.8 times longer in its absence. For repeated displays, the efficiency of the scan paths additionally increased to a score of 2.3 times the most direct distance (due to contextual cueing) whereas the scan pattern ratio for novel and repeated displays was virtually identical for search with a scotoma. Together, these data indicate that the need to adapt exploration to maximize visual sampling in the periphery led to less efficient search with the scotoma. This less efficient search is characterized by an equal number of fixations that were placed less efficiently than in unimpaired search.

The inefficient top-down controlled search mode may be one possible reason why we did not observe search facilitation for repeated displays in search with a scotoma. A reduction of search facilitation for top-down controlled search strategies in comparison with more stimulus-guided search has been described previously (Lleras and von Mühlenen, 2004). Future experiments with longer training, leading to more automatic search with simulated scotomata, may address this question. The same issue is of course highly relevant for the question whether patients with AMD in whom the foveal vision impairment develops gradually, thereby leaving time to adapt visual search behavior, will also lack a search facilitation in repeated displays.

It has been shown that search facilitation in repeated displays depends on attending to the repeated display items (Jiang and Chun, 2001). Similarly, filling visuospatial working memory capacity by a concurrent task eliminated the search advantage in repeated displays (Manginelli et al., 2011). These demands on attentional and working memory capacity were primarily observed when previously learned templates needed to be retrieved in order to speed up search in repeated displays, while learning of repeated displays itself was still observed when attention or visual working memory was reduced by secondary demands (Jiang and Leung, 2005; Vickery et al., 2010 ). Thus it is likely that the presence of a scotoma, by impairing the free exploration of a search display (or other environment) may make the match between search templates from long-term memory and the current search display more difficult, thereby reducing or eliminating the search advantage for repeated displays observed in non-scotomatous participants. Similarly, Fiske and Sanocki (2011) presented evidence for a reduction of the efficiency of attentional guidance in repeated displays by crowding. Whereas Fiske and Sanocki increased crowding by reducing the spacing between search items, using peripheral vision for search also increases crowding, because the minimum distance for uncrowded viewing increases toward the periphery of the visual field (Pelli and Tillman, 2008). Future research may address the question whether the interference observed for contextual cueing in the present experiment may also be observed for other kinds of cueing, such as probability cueing of the target location (Geng and Behrmann, 2002) or distinct priming of target or distractors in visual search (Kristjánsson and Driver, 2008).

Brady and Chun (2007) proposed a model of contextual cueing assuming that mostly the local context information close to the target location is learned in repeated displays, suggesting that a "snapshot" of the target and its surrounding distractors is made when the target is detected by the observer. Nevertheless, they also observed a contribution of global regularities to contextual cueing. van Asselen and Castelo-Branco (2009) examined the contribution of peripheral vision to contextual cueing. In their study, participants were instructed to fixate centrally throughout display presentation. Fixation was monitored by gaze tracking. The search items in their study were presented at eccentricities between $2.4^{\circ}$ and $4.1^{\circ}$. They observed a robust contextual cueing effect which was maintained during a period of 10 days after the initial learning experiment. The authors concluded that contextual learning can take place in peripheral vision and is thus not dependent on high-acuity foveal resolution. In contrast, we did not observe facilitation for repeated displays in the scotoma condition in which observers were forced to use peripheral vision for target detection.

Two important differences between the study of van Asselen and Castelo-Branco (2009) and our data have to be considered. First, the stimulus positions used by van Asselen and CasteloBranco (2009) fell approximately into the area covered by the scotoma in our study, that covered an area of $3.5^{\circ}\left(4.5^{\circ}\right.$ including the Gaussian fading at the border which additionally degraded the visual input to some extent). Thus it may be argued, that the presently used scotoma size impaired the parafoveal region which might be crucial for the learning of the statistical associations between target and surrounding distractor stimuli in repeated displays. This, however, appears unlikely, because previous data have shown that contextual cueing can occur across much larger distances as long as there is no interference of nonrepeated distractors placed between predictive distractors and the target (Olson and Chun, 2002). A second important difference to the study by van Asselen and Castelo-Branco (2009) concerns the use of eye-movements. Their observers were forced to inhibit eye-movements and perceived the complete search display peripherally "at a glance." Thus, the perceived spatial relations between items remained static throughout the search trial, which could potentially contribute to learning of the context information. In contrast, eye-movements were necessary in our study due to the increased search difficulty introduced by making the distractors look more similar to the target. The observed number of fixations clearly shows that our observers did indeed move their eyes in order to find the target. As a consequence of those eye-movements, the scotoma dynamically changed the appearance of the search displays by rendering parts of the stimulus arrays invisible to the observer. Thus, the memory templates for the repeated search displays may have been more patchy in the scotoma condition as the 
observers were never able to perceive the complete stimulus arrays at one glance which, in turn, might lead to the observed lack of contextual cueing. It would be interesting to see if contextual cueing was intact using smaller scotoma sizes which may still impair the planned execution of eye-movements and lead to inefficient scan paths compared to unimpaired search but allow for a more holistic peripheral processing of the search displays. Taken together the results of van Asselen and Castelo-Branco (2009) and our results might suggest that an optimal search strategy with a scotoma leading to the development of contextual cueing might be to reduce eye-movements and covertly shift attention across the stimuli perceived in the periphery. This issue might be addressed comparing the relation between the magnitude of contextual learning and eye-movement patterns in trained and untrained search with the scotoma.

Interestingly, we found a positive correlation between the awareness of context repetition and the magnitude of contextual cueing in the scotoma condition. Whereas many studies pointed out the implicit nature of contextual cueing (Chun and Jiang, 1998; Chun and Phelps, 1999; Howard et al., 2004; Manginelli and Pollmann, 2009), Smyth and Shanks (2008) and Geyer et al. (2010a) provided evidence that explicit memory of context repetition may at least be present for a subset of repeated displays, a fact that may have been overlooked in previous studies because explicit recognition tests had less statistical power than the implicit memory measure. In the current experiments, the explicit recognition test carried out immediately after the search task yielded evidence for at least partly explicit learning in unimpaired search, whereas no such evidence was obtained in the scotoma experiment. As in previous studies (Smyth and Shanks, 2008; Geyer et al., 2010a), individual explicit memory scores were not correlated with the size of search facilitation in unimpaired search. In contrast, there was a significant correlation in the scotoma experiment. Taken together, these results may indicate that in our experiment, observers in both viewing conditions became aware of repeated search arrays which facilitated target detection. If repeated displays gain access to conscious perception, this might be a sign that the (implicit) memory traces of the repeated layouts gain so much strength that observers eventually become aware of the repetition as more cognitive resources might be available to encode the exact positions of the stimuli during "automatic" search. In the scotoma condition, aware observers might thus have improved their search strategies so efficiently that they were able to build up robust memory representations of the repeated search arrays whereas in the control condition visual search was much less demanding such that strong associations between target and distractor positions may have evolved in all observers, leading to significant awareness on a group level. Until today, there is no evidence that explicitly recognized search layouts improve contextual cueing effects. Future studies might address this issue with more elaborated recognition tests, e.g., one could ask the subjects to submit confidence ratings on whether they have seen the display before and analyze the results in terms of more sophisticated measures of signal detection theory. The aim of the present study was primarily to investigate whether incidental (i.e., noninstructed) learning of spatial configurations could be used for more efficient visual search in repeated search environments.
Whether this incidental learning occurs in a truly implicit way (i.e., without awareness) is not easy to assess (Merikle and Reingold, 1992; Wiens, 2007). Although interesting in itself it was not our prime concern to investigate this issue because we wanted to know whether patients with a central scotoma, e.g., due to AMD, might be impaired in visual search in repeated environments at all.

Another important aspect that needs to be addressed in future studies is whether the learning of spatial configurations itself is impaired in search with a central scotoma or if the use of learned configurations is impaired, e.g., due to the need for top-down controlled search suggested by our data. On the one hand, the importance of fixating (and thereby attending) an object for later explicit recall has been clearly demonstrated (Hollingworth, 2006). On the other hand, it has been shown that incidental context learning in visual search may proceed in the absence of focused attention on the repeated search items (Jiang and Leung, 2005). Thus it remains an open question whether the presence of a central scotoma interferes with learning of context cues. Future studies may address this question by removing the scotoma after a learning phase with scotoma, something that obviously cannot be done with natural scotomata.

The investigation of scotoma effects on contextual cueing was inspired by the presence of foveal viewing deficits in patients with AMD. The present results show that AMD patients may suffer from deficits in contextual cueing that may complicate their exploration of the environment in addition to their low-level visual impairments. There are, however, several caveats before the present results can be taken to reflect contextual cueing in AMD. On the one hand, our data present a purer view on the effect of a scotoma on contextual cueing than the performance of AMD patients, which may present with additional visual or cognitive impairments. On the other hand, patients may develop adaptive strategies over time that may help them to regain contextual cueing to some degree.

To our knowledge, this is the first demonstration that the presence of a scotoma impairs contextual cueing in visual search. The gaze-contingent scotoma simulation prevented participants to use learned spatial configurations to improve the efficiency of visual search. The effect of the simulated scotoma was specific for visual learning of repeated displays. In contrast, unspecific procedural learning over the experimental session led to an equal acceleration of search with and without scotoma, independent of repetition or novelty of the displays. This specific lack of a search facilitation in repeated displays was not due to an increased number of fixations but rather due to a less efficient scan path used to search the display. Future studies are needed to investigate the causes of this deficit, which may be interference with contextual learning itself or interference with the later use of previously learned contexts, and finally how training can improve visual learning and its use for efficient visual search with a central scotoma.

\section{ACKNOWLEDGMENTS}

This work was supported by grants of the Deutsche Forschungsgemeinschaft (PO548/6-2 and 8-1). We thank Ina Pohl for assistance in data acquisition. 


\section{REFERENCES}

Bedell, H. E., Tong, J., Woo, S. Y., House, J. R., and Nguyen, T. (2009). Orientation discrimination with macular changes associated with early AMD. Optom. Vis. Sci. 86, 485-491.

Bertera, J. H. (1988). The effect of simulated scotomas on visual search in normal subjects. Invest. Ophthalmol. Vis. Sci. 29, 470-475.

Brady, T. F., and Chun, M. M. (2007). Spatial constraints on learning in visual search: modeling contextual cuing. Exp. Psychol. Hum. Percept. Perform. 33, 798-815.

Brainard, D. H. (1997). The psychophysics toolbox. Spat. Vis. 10, 433-436.

Brockmole, J. R., and Henderson, J. M. (2006). Recognition and attention guidance during contextual cueing in real-world scenes: evidence from eye movements. Q. J. Exp. Psychol. 59, $1177-1187$.

Chun, M. M., and Jiang, Y. (1998). Contextual cueing: implicit learning and memory of visual context guides spatial attention. Cogn. Psychol. 36, 28-71.

Chun, M. M., and Jiang, Y. (2003). Implicit, long-term spatial contextual memory. J. Exp. Psychol. Learn. Mem. Cogn. 29, 224-234.

Chun, M. M., and Phelps, E. A. (1999). Memory deficits for implicit contextual information in amnesic subjects with hippocampal damage. Nat. Neurosci. 2, 844-847.

Cornelissen, F. W., Bruin, K. J., and Kooijman, A. C. (2005). The influence of artificial scotomas on eye movements during visual search. Optom. Vis. Sci. 82, 27-35.

Deubel, H., and Schneider, W. X. (1996). Saccade target selection and object recognition: evidence for a common attentional mechanism. Vis. Res. 36, 1827-1837.

Fiske, S., and Sanocki, T. (2011). Does crowding obscure the presence of attentional guidance in contextual cueing? J. Vis. 11, 1296 (Abstract).

Geng, J. J., and Behrmann, M. (2002). Probability cuing of target location facilitates visual search implicitly in normal participants and patients with hemispatial neglect. Psychol. Sci. 13, 520-525.

Geyer, T., Shi, Z., and Müller, H. J. (2010a). Contextual cueing in multiconjunction visual search is dependent on color- and configurationbased intertrial contingencies. J. Exp.
Psychol. Hum. Percept. Perform. 36, 515-532.

Geyer, T., Zehetleitner, M., and Müller, H. J. (2010b). Contextual cueing of pop-out visual search: when context guides the deployment of attention. J. Vis. 10, 1-11.

Hoffman, J. E., and Subramaniam, B. (1995). The role of visual attention in saccadic eye movements. Percept. Psychophys. 57, 787-795.

Hollingworth, A. (2006). Visual memory for natural scenes: evidence from change detection and visual search. Vis. Cogn. 14, 781-807.

Howard, J. H., Howard, D. V., Dennis, N. A., Yankovich, H., and Vaidya, C. J. (2004). Implicit spatial contextual learning in healthy aging. Neuropsychology 18, 124-134.

Jiang, Y., and Chun, M. M. (2001). Selective attention modulates implicit learning. Q. J. Exp. Psychol. A 54, 1105-1124.

Jiang, Y., and Leung, A. W. (2005). Implicit learning of ignored visual context. Psychon. Bull. Rev. 12, 100-106.

Kristjánsson, A., and Campana, G. (2010). Where perception meets memory: a review of repetition priming in visual search tasks. Atten. Percept. Psychophys. 72, 5-18.

Kristiánsson, A., and Driver, J. (2008). Priming in visual search: separating the effects of target repetition, distractor repetition and role-reversal. Vis. Res. 48, 1217-1232.

Kunar, M. A., Flusberg, S., Horowitz, T. S., and Wolfe, J. M. (2007). Does contextual cuing guide the deployment of attention? J. Exp. Psychol. Hum. Percept. Perform. 33, 816-828.

Kunar, M. A., Flusberg, S. J., and Wolfe, J. M. (2008). Time to guide: evidence for delayed attentional guidance in contextual cueing. Vis. Cogn. 16, 804-825.

Lavie, N., Hirst, A., De Fockert, J. W., and Viding, E. (2004). Load theory of selective attention and cognitive control. J. Exp. Psychol. Gen. 133, 339-354.

Lindblad, A. S., Lloyd, P. C., Clemons, T. E., Gensler, G. R., Ferris, F. L. III, Klein, M. L., and Armstrong, J. R. (2009). Change in area of geographic atrophy in the age-related eye disease study: AREDS report number 26. Arch. Ophthalmol. 127, 1168-1174.

Lleras, A., and von Mühlenen, A. (2004). Spatial context and top-down strategies in visual search. Spat. Vis. 17, 465-482.
Manginelli, A. A., Geringswald, F., and Pollmann, S. (2011). Visual search facilitation in repeated displays depends on visuospatial working memory. Exp. Psychol. 18, 1-8.

Manginelli, A. A., and Pollmann, S. (2009). Misleading contextual cues: how do they affect visual search? Psychol. Res. 73, 212-221.

Merikle, P., and Reingold, E. (1992). "Measuring unconscious perceptual processes," in Perception without Awareness, eds R. Bornstein and T. Pittman (New York: Guilford), 55-80.

Miellet, S., Zhou, X., He, L., Rodger, H. and Caldara, R. (2010). Investigating cultural diversity for extrafoveal information use in visual scenes. $J$. Vis. 10, 21.

Murphy, K., and Foley-Fischer, J. A. (1988). Visual search with non-foveal vision. Ophthalmic Physiol. Opt. 8, 345-348.

Neelam, K., Nolan, J., Chakravarthy, U., and Beatty, S. (2009). Psychophysical function in age-related maculopathy. Surv. Ophthalmol. 54, 167-210.

Nyström, M., and Holmqvist, K. (2010). An adaptive algorithm for fixation, saccade, and glissade detection in eyetracking data. Behav. Res. Methods 42, 188-204.

Olson, I. R., and Chun, M. M. (2002) Perceptual constraints on implicit learning of spatial context. Vis. Cogn. 9, 273-302.

Pelli, D. G. (1997). The videotoolbox software for visual psychophysics: transforming numbers into movies. Spat. Vis. 10, 437-442.

Pelli, D. G., and Tillman, K. A (2008). The uncrowded window of object recognition. Nat. Neurosci. 11, 1129-1135.

Peterson, M. S., and Kramer, A. F. (2001). Attentional guidance of the eyes by contextual information and abrupt onset. Percept. Psychophys. 63, 1239-1249.

Qiu, F., and Leat, S. J. (2009). Functional deficits in early stage age-related maculopathy. Clin. Exp. Optom. 92, 90-98.

Rayner, K., and Bertera, J. H. (1979). Reading without a fovea. Science 206, 468.

Savitzky, A., and Golay, M. J. E. (1964). Smoothing and differentiation of data by simplified least squares procedures. Anal. Chem. 36, 1627-1639.

Shen, J., Reingold, E. M., Pomplun, M., and Williams, D. E. (2003). "Saccadic selectivity during visual search: the influence of central processing difficulty," in The Mind's Eye: Cognitive and Applied Aspects of Eye Movement Research, eds J. Hyona, R. Radach, and H. Deubel (Amsterdam: Elsevier), $65-88$.

Smyth, A. C., and Shanks, D. R. (2008). Awareness in contextual cuing with extended and concurrent explicit tests. Mem. Cogn. 36, 403-415.

Timberlake, G. T., Mainster, M. A., and Peli, E. (1986). Reading with a macular scotoma. I. Retinal location of scotoma and fixation area Invest. Ophthalmol. Vis. Sci. 27, 1137-1147.

Tseng, Y.-C., and Li, C.-S. R. M. (2004). Oculomotor correlates of contextguided learning in visual search. Percept. Psychophys. 66, 1363-1378.

van Asselen, M., and Castelo-Branco, M. (2009). The role of peripheral vision in implicit contextual cuing. Atten Percept. Psychophys. 71, 76-81.

Vickery, T. J., Sussman, R. S., and Jiang, Y. V. (2010). Spatial context learning survives interference from working memory load. J. Exp. Psychol. Hum Percept. Perform. 36, 1358-1371.

Wang, Y.-Z., Wilson, E., Locke, K. G., and Edwards, A. O. (2002). Shape discrimination in age-related macular degeneration. Invest. Ophthalmol. Vis. Sci. 43, 2055-2062.

Wiens, S. (2007). Concepts of visual consciousness and their measurement. Adv. Cogn. Psychol. 3, 349-359.

Conflict of Interest Statement: The authors declare that the research was conducted in the absence of any commercial or financial relationships that could be construed as a potential conflict of interest.

Received: 09 February 2012; accepted: 26 April 2012; published online: 11 May 2012.

Citation: Geringswald F, Baumgartner F and Pollmann S (2012) Simulated loss of foveal vision eliminates visual search advantage in repeated displays. Front. Hum. Neurosci. 6:134. doi: 10.3389/ fnhum.2012.00134

Copyright (๑) 2012 Geringswald, Baumgartner and Pollmann. This is an openaccess article distributed under the terms of the Creative Commons Attribution Non Commercial License, which permits non-commercial use, distribution, and reproduction in other forums, provided the original authors and source are credited. 